\title{
El acontecimiento como enigma y huella
}

\author{
Q Graciela Ralón \\ Universidad Nacional de San Martín, Argentina
}

Recibido el 02/og/2020. Aceptado el 20/11/2020.

\begin{abstract}
Resumen
El propósito de este trabajo apunta, en primer lugar, a dilucidar en la Fenomenología de la percepción, el marco a partir del cual el autor comprende la historia y, en particular, el acontecimiento histórico. Según mi opinión, la descripción de este marco, de fuerte carácter existencialista, nos permitirá, en un segundo momento, avanzar hacia los rasgos específicos del acontecimiento histórico: singularidad, generalidad y contingencia, que ofrecen la clave para comprender en qué medida el acontecimiento es, desde la mirada merleau-pontyana, enigma y huella. Finalmente, una mirada prospectiva de la obra del autor nos permitirá ubicar la posición merleau-pontyana en el contexto de la discusión actual.

Palabras clave: acontecimiento, huella, enigma, contingencia, historicidad.
\end{abstract}

\section{The event as enigma and trace}

\begin{abstract}
The purpose of this article is, first of all, to elucidate in the Phenomenology ofperception, the framework from which the author understands history and, in particular, the historical event. It is argued that the description of this framework, which has a strong existentialist character, will allow us, in a second moment, to advance towards the specific features of the historical event: singularity, generality, and contingency, which offer the key to understand the extent in which the event is, from Merleau-Ponty's point of view, an enigma and a trace. Finally, a prospective look at the author's work will allow us to locate the position of Merleau-Ponty in the context of the current discussion.
\end{abstract}

Keywords: event, trace, enigma, contingency, historicity. 


\section{Introducción}

En la obra titulada El renacimiento del acontecimiento. Un desafio para el historiador: entre Fénixy Esfinge, François Dosse pone a prueba, en el conjunto de las ciencias humanas, la noción de acontecimiento con la finalidad de medir su fecundidad potencial y su valor heurístico. A partir de la acepción dada por Michel de Certeau "el acontecimiento es lo que llega" (de Certeau, 1994: 51), éste es interpretado por un desplazamiento que no se remonta a la búsqueda de sus causas, sino que, por el contrario, retrocede hacia sus huellas (cf. Dosse, 2010: 11). El objeto de la investigación de Dosse es buscar las claves de comprensión de la era actual que está atravesada por una relación con la historicidad que atañe a su carácter acontecial (événementialiasation). No se trata, pues, de un retorno hacia lo factual sino del nacimiento de una nueva mirada sobre el acontecimiento ${ }^{1}$. Según Dosse

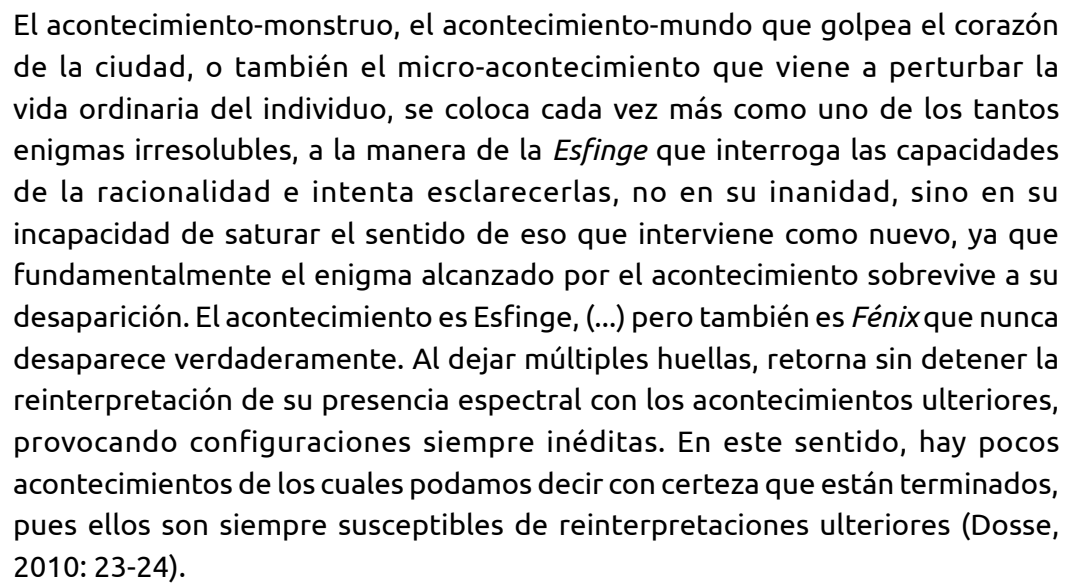

Estos dos aspectos del acontecimiento, su carácter enigmático y la posibilidad de su reanudación a través de sus huellas, están presentes en las reflexiones de MerleauPonty acerca de la historicidad de la vida en todas sus expresiones. Si bien no se encuentra en su obra una reflexión sistemática y explícita sobre la noción de acontecimiento, sin embargo, no deja de llamar la atención que las reflexiones acerca de la historia, especialmente en los escritos contemporáneos a la Fenomenología de la percepción, giran en torno a la comprensión de la historia como dimensión esencial de la existencia. En las reflexiones llevadas a cabo ya desde esta época, puede advertirse que la historia es, a la vez, "génesis de sentido", lo cual implica la posibilidad de reasunciones y nuevas interpretaciones; y de enigma, en el sentido en que que, algunas veces, en el amor, en la plenitud de la obra o en las acciones políticas, los hombres se reúnen y los acontecimientos responden a su voluntad: "Algunas veces se da este abrazo, esta luz, este momento de victoria o, como dice la María de Hemingway, esta gloria que lo borra todo" (Merleau-Ponty, 1963: 330).

Según mi opinión, en Fenomenología de la percepción las reflexiones acerca del mundo social, la libertad o la temporalidad sientan los pilares y constituyen el horizonte a partir del cual serán pensados los rasgos centrales del acontecimiento en los años venideros: singularidad, contingencia, ambigüedad. Estas cuestiones que atañen a la vida en todas sus manifestaciones y que, por otra parte, distinguen al acontecimiento

\footnotetext{
1 Según Françoise Dosse, este retorno no tiene nada que ver con la concepción restrictiva de la escuela histórica metódica del siglo XIX y, aclara que: “(...) Más que un retorno, vivimos un renacimiento o un regreso de la diferencia” (Dosse, 2010: 24). La atención prestada a esta nueva perspectiva, esto es, a las huellas y sus transformaciones evita caer en el dilema entre la corta y larga duración, planteada por la Escuela de los Anales. "Al mismo tiempo Esfinge y Fénix el acontecimiento escapa a toda pretensión de reduccionismo" (Ibid., 39-40).
} 
histórico, pueden ser leídos a lo largo de todos los capítulos de la obra de $1945 . \mathrm{Me}$ centraré aquí, sin embargo, principalmente en "El otro y el mundo humano" y "La libertad". Tomaré, por otra parte, los trabajos comprendidos entre 1945 y 1949 de los que pueden extraerse ciertos elementos que refuerzan las tesis de Fenomenología de la percepción, y contribuyen a pensar la vida histórica como una trama que se desarrolla según un orden, pero que implica "un juego en el sistema" en virtud del cual los acontecimientos no están rigurosamente ligados. Esto significa que la dialéctica de la historia puede "encaminarse o desviarse" sin llegar a resolver todos los acontecimientos que aparecen. La historia lleva consigo significaciones esbozadas "como un interlocutor distraído deja que el debate se desvíe, se olvida en el camino de los datos del problema [...]" (Merleau-Ponty, 1955: 40).

Si bien en Fenomenología Merleau-Ponty no ha llevado a cabo una reflexión sistemática de la historia como la que presenta, por ejemplo, en el curso titulado "Materiales para una teoría de la historia", la contingencia de la vida humana con la que MerleauPonty caracteriza, principalmente, los hechos de carácter histórico, nos permitirá ver, de todos modos, que la efectuación de un acontecimiento es la realización de una "posibilidad entre otras", y que en ello reside, precisamente, su carácter enigmático. Teniendo en cuenta este trasfondo, intentaremos, en primer lugar, esbozar el marco a partir del cual el autor comprende, en Fenomenología de la percepción, la historia y, en particular, el acontecimiento histórico. Según mi opinión, la descripción de este marco, de fuerte carácter existencialista, nos permitirá avanzar hacia los rasgos específicos del acontecimiento histórico, esto es, su singularidad, su generalidad y su contingencia, rasgos que ofrecen la clave para comprender en qué medida el acontecimiento es, desde la mirada merleau-pontyana, enigma y huella. Finalmente, una mirada prospectiva de la obra del autor nos permitirá ubicar la posición merleau-pontyana en el contexto de la discusión actual.

\section{La comprensión de la historia como "génesis de sentido"}

Intentaremos en esta primera parte poner de manifiesto el marco descriptivo que ofrece Fenomenología de la percepción con la finalidad de comprender la historia como génesis de sentido. El entramado situación-libertad y la convergencia de diferentes órdenes, que confluyen en la interpretación de los acontecimientos, constituyen los núcleos centrales de dicha comprensión.

\subsection{Entramado situación-libertad}

Lo primero que hay que señalar es la pertenencia del sujeto histórico a un mundo social, el cual no es vivido por él como un objeto que se encuentra a su alrededor o que puede contemplar desde afuera para con posterioridad otorgarle una significación. El mundo social es "[...] el campo permanente o dimensión de la existencia: yo puedo apartarme, pero no cesar de estar situado en relación con él" (Merleau-Ponty, 1945: 415). No sería posible una conciencia objetiva y científica de las civilizaciones, si el sujeto no tuviera con ellos, a través de su mundo social y cultural, una comunicación, "[...] si no estuvieran instalados como otros tantos individuos por conocer, indeterminados pero preexistentes, si no encontrara en mi vida las estructuras fundamentales de la historia" (Merleau-Ponty, 1945: 415Así se advierte que, cuando la constelación natural o social se cristaliza en una situación, es decir, desde el momento en que existimos, la conciencia no puede ser vista como una conciencia pura: "Hay un sentido autóctono del mundo que se constituye en el comercio de nuestra existencia encarnada con él y que forma el suelo de toda Sinngebung respecto de las decisiones" (Merleau-Ponty, 1945: 503). Por otra parte, las estructuras históricas al igual que las estructuras perceptivas son ambiguas, lo cual significa que pueden ser alteradas a través de las valoraciones espontáneas que se hacen visibles en las iniciativas humanas: 
Yo soy una estructura psicológica e histórica. He recibido con la existencia una manera de existir, un estilo. Todas mis acciones y mis pensamientos están en relación con esta estructura [...]. Y, sin embargo, soy libre, no a pesar de o más acá de estas motivaciones, sino por su medio. Pues esta vida significativa, esta cierta significación de la naturaleza y de la historia que soy no limita mi acceso al mundo; es, por el contrario, mi medio de comunicarme con él. (Merleau-Ponty, 1945: 519).

Estas afirmaciones nos conducen a considerar la noción de existencia como una noción paradójica que implica una tensión entre libertad y situación. La existencia es “[...] una actividad dada a sí misma en una situación natural e histórica, y tan imposible es abstraerse de como reducirse a ella" (Merleau-Ponty, 1963: 237). Desde esta perspectiva se enfatiza tanto el carácter situado de la vida humana como la posibilidad de autonomía a partir de la dependencia. No estamos determinados y, sin embargo, el sentido total de lo que hacemos no se reduce a nuestros proyectos personales y conscientes. De esta manera, la situación existencial se convierte en el fundamento del devenir histórico. La historia se presenta como la consecuencia del modo en que los hombres responden a su situación personal y colectiva y la retoman en sus proyectos expresivos. Es necesario comprender, como veremos, que nuestra existencia emerge de un fondo de generalidad que comprende otras decisiones y otros proyectos: "[...] soy todo cuanto veo, soy un campo intersubjetivo, no a pesar de mi cuerpo y mi situación histórica, sino, por el contrario, por ser este cuerpo y esta situación, y todo lo demás a través de ellos" (Merleau-Ponty, 1945: 515).

Con la noción de situación Merleau-Ponty quiere poner de manifiesto que cada existencia es la revelación de una configuración dada, esto es, de una situación física e histórica llamada a expresarse: "cada sujeto encarnado es como un registro abierto del que no se sabe lo que se inscribirá ahí [...], pero que una vez aparecido, no podrá dejar de decir poco o mucho, de tener una historia o un sentido" (Merleau-Ponty, 2000: 41). Por otra parte, en la situación se conjugan tres instancias, que Merleau-Ponty expresa de la siguiente manera: “[...] no pienso ni según lo verdadero solamente, ni según yo solo, ni según el otro solamente, puesto que cada uno tiene necesidad de los otros dos [...]" (Merleau-Ponty, 1953: 40).

A través de la descripción de cómo surge la noción de conciencia de clase, el fenomenólogo francés ilustra cómo la libertad no destruye la situación puesto que ésta solo tiene sentido pensada desde la situación. Nuestras decisiones responden a un contexto y deben ser leídas e interpretadas a partir de él. Para el pensamiento objetivo la conciencia de clase es el efecto mecánico de la pertenencia a un estrato social. Por el contrario, para una posición idealista la pertenencia al proletariado, por ejemplo, se reduciría a una toma de conciencia independiente de la historia personal y cultural que nos constituye. "Al pensamiento objetivo, que inserta al sujeto en la red del determinismo, oponemos la reflexión idealista que hace reposar el determinismo sobre la actividad constituyente del sujeto" (Merleau-Ponty, 1945: 506). Frente a estos puntos de vista extremos, Merleau-Ponty sostiene que lo primero con lo que el hombre se encuentra es su modo de existir. La transformación de esta existencia vivida en una existencia consciente implicará que la decisión deje de ser abstracta y adquiera una realidad histórica. Con otras palabras, es el modo de comunicarnos y relacionarnos con el mundo lo que motiva un proyecto revolucionario o un proyecto conservador. Así, el proyecto existencial es "[...] la polarización de una vida hacia un fin determinado-indeterminado del que no se tiene ninguna representación y que solo se reconoce en el momento de alcanzarlo" (Merleau-Ponty, 1945: 509). Es necesario comprender conjuntamente que, si nada solicitara mi libertad, la historia no entrañaría ninguna estructura: "[...] damos su sentido a la historia, pero no sin que ella nos lo proponga” (Merleau-Ponty, 1945: 513). 


\subsection{La conjunción de diferentes órdenes}

En el "Prólogo" a la Fenomenología de la percepción, Merleau-Ponty se pregunta si se ha de comprender la historia a partir de la ideología, la religión o la economía o la doctrina de un autor por su contenido, por su psicología o por las situaciones vividas por él. Frente a estos interrogantes, responde que "[...] es necesario comprender de todas las maneras a la vez, todo tiene un sentido, encontramos bajo todas las relaciones la misma estructura de ser" (Merleau-Ponty, 1945: XIV). Del mismo modo que las diferentes partes del cuerpo se implican en la unidad de un movimiento, en el caso de los acontecimientos sociales diversos factores confluyen y se entrelazan en una unidad de significación; $y$, al igual que hay una diversidad de posibilidades de movimiento, esta red de significaciones es abierta y deja entrever diversas significaciones. En esta pluralidad de aspectos reside el carácter ambiguo de los hechos históricos y lo que impide, a la vez, asignar a la historia un sentido único. Ya desde la época de Fenomenología de la percepción, Merleau-Ponty interpretaba la historia como una "génesis de sentido" (Sinngenesis) que a pesar de sus avatares enseña lo que los hechos "quieren decir" y finaliza por convertir los azares en razón. Todo se revela como "un solo drama" del que no se sabe si habrá un desenlace posible, pero al cual no podemos renunciar porque "estamos condenados al sentido". Lo que diferencia la concepción existencial de la historia del materialismo o del espiritualismo se encuentra en la multiplicidad de significados que acompaña a toda acción humana (Merleau-Ponty, 1945: 202). La historia efectúa un intercambio de todos los órdenes de actividad y ninguno de ellos puede ser considerado como una causa única. Desde esta perspectiva, la economía, por ejemplo, no puede ser interpretada como un ciclo cerrado de fenómenos objetivos, sino como "[...] una confrontación de las fuerzas productivas y de las formas de producción que solo llega a su término cuando las primeras salen de su anonimato, toman conciencia de ellas mismas, y llegan así a ser capaces dar forma al porvenir" (Merleau-Ponty, 1945: 199). Esta toma de conciencia es un fenómeno cultural y es lo que permite introducir en la trama de la historia motivaciones psicológicas hasta tal punto que “[...] la economía se encuentra reintegrada a la historia antes que la historia reducida a la economía" (Merleau-Ponty, 1945: 200). Desde el momento en que la economía se encuentra arraigada en la existencia social y concreta de una sociedad, no es posible concebir una causalidad económica pura, ni tampoco negarles a las situaciones económicas su poder de motivación. Entre las denominadas "infra" " "super" estructuras no hay una explicación causal y mecánica porque sobre la misma base objetiva pueden edificarse diferentes comportamientos.

\section{Rasgos que caracterizan al acontecimiento histórico: singulari- dad, anonimato, y contingencia.}

\subsection{La singularidad del acontecimiento}

En el escrito presentado para la candidatura al Collège de France, el autor realiza una presentación de los resultados alcanzados en sus dos primeras obras y a partir de ellos señala el rumbo que seguirán sus próximas investigaciones. Antes de describir ese itinerario Merleau-Ponty tiene presente que:

El sujeto de la percepción no es ese pensador absoluto, funciona por aplicación de un pacto pasado entre nuestro nacimiento, nuestro cuerpo y el mundo, entre nosotros mismos y nuestro cuerpo; es como un nacimiento continuado, aquél a quien una situación física e histórica ha sido dada a manifestar, y lo es a cada instante de nuevo (Merleau-Ponty, 2000: 40-41. Traducción nuestra).

Desde el momento en que el nacimiento abre un campo de dimensiones durables queda abierta la posibilidad de una historia tanto personal como pública. Con el acontecimiento del nacimiento aparece un ser que no se define de entrada ni como una serie de 
estados de conciencia ni como una mónada, sino como un campo de experiencias: "En la casa donde un niño nace, todos los objetos cambian de sentido, ellos se ponen a esperar de él un tratamiento todavía indeterminado, alguien otro aparece ahí, una nueva historia, breve o larga, viene a ser fundada, un nuevo registro está abierto" (Merleau-Ponty, 1945: 466). Así, el acontecimiento del nacimiento no es solo el comienzo fechado de una vida, sino la apertura de una experiencia perceptiva que no cesará de continuarse. Es un "acontecimiento" o un "acontecimiento trascendental" (Thierry, 2005: 145), esto es, un acontecimiento que es condición de posibilidad de otros acontecimientos. Años más tarde, en el curso dedicado a la noción de institución, Merleau-Ponty vuelve a confirmar el sentido del nacimiento. Al caracterizar la noción de institución, explícitamente, desde la fenomenología genética afirma que: "El nacimiento (no es un acto) de constitución sino institución de un por-venir" (Merleau-Ponty, 2003: 38. Traducción nuestra). La institución solo tiene sentido para la conciencia porque para ella todo es instituido (es decir: "puesto") y, más adelante, agrega: "Constituir [...] es casi lo contrario de instituir: lo instituido tiene sentido sin mí, lo constituido no tiene sentido más que para mí y para el yo de ese instante" (Merleau-Ponty, 2000: 37).

Cabe señalar que las discusiones fenomenológicas actuales acerca del acontecimiento, coinciden en destacar que el carácter no-elegido del nacimiento, lo convierte en un "protoacontecimiento" y, a su vez, su carácter sorpresivo, le otorga la condición de lo inesperado: "hay una sorpresa en nosotros en relación con nuestro nacimiento. Es la sorpresa permanente de nacer que es constitutiva de nuestro ser" (Dastur, 2000, 186). Por otra parte, F. Dastur señala que, este carácter sorpresivo se repetirá en todo nuevo acontecimiento, con palabras de la autora:

En cada nuevo acontecimiento hay una repetición del protoacontecimiento del nacimiento. Es como si volviéramos a experimentar, en un nuevo acontecimiento la novedad radical de lo que sucede por Dprimera vez', así como la imposibilidad de coincidir con el acontecimiento mismo, que en su repentina aparición desconecta el pasado del futuro" (Dastur, 2000: 186).

Si bien compartimos con la posición de Dastur el carácter sorpresivo e inesperado del acontecimiento, como veremos más adelante, no creemos que esta no coincidencia desconecte el pasado del futuro. La novedad no le quita al acontecimiento su historicidad.

\subsection{La generalidad del acontecimiento}

La generalidad que envuelve a la existencia humana no solo le ofrece al sujeto un conjunto de capacidades ligadas al saber hacer sino que contempla también las experiencias realizadas por otros hombres que hacen del mundo natural un mundo social. Así: "Nosotros reconocemos, alrededor de nuestras iniciativas y de ese proyecto rigurosamente individual que somos, una zona de existencia generalizada y de proyectos ya hechos, de significaciones que se deslizan (trainent) entre nosotros y las cosas [...]" (Merleau-Ponty, 1945: 513). Entre las formas generalizadas de existencia y la vida de cada uno se da una relación a la vez centrípeta y centrífuga por la cual esas estructuras anónimas y la existencia individual son a la vez activas y pasivas: "Hay un momento en que el sentido que se dibujaba en el Se (On), y que solo era algo posible, inconsistente, amenazado por la contingencia de la historia, es retomado por un individuo" (Merleau-Ponty, 1945: 513). Porque el mundo está ya constituido, nuestra libertad no puede ser total, sino que tiene un sostén y un medio para realizarse, tanto en el estilo que continúa como en las motivaciones que recibe y, por la misma razón, la libertad no puede ser nula y estamos abiertos a nuevas posibilidades. Así, la historia nos envuelve y nos realizamos a través de esta inherencia de ella en nosotros, no por medio de una creación absoluta sino de un deslizamiento o desviación de sentido. El sentido de los acontecimientos no proviene ni de una idea que los produce ni del resultado fortuito de los mismos. Hay una existencia funcional y generalizada que 
convierte a los sujetos en sujetos históricos; por lo tanto, hay que reconocer que las iniciativas personales emergen de esta existencia generalizada en la que han sedimentado otras iniciativas y otros proyectos, significaciones que la conciencia no extrae a partir de la nada sino que se forman en el trato con esas iniciativas y esos proyectos.

Situación, sentido de la historia y verdad histórica, son tres maneras de decir lo mismo y solo pueden darse en la medida en que nuestro ser del mundo es portador de un doble anonimato. Los acontecimientos históricos son el fruto de un proyecto concreto, pero aparecen diseñados con anterioridad en la coexistencia social y en lo impersonal. Con otras palabras, hay una existencia adquirida que contiene lo nuevo "en estado de huella o de horizonte" (Merleau-Ponty, 1969: 183) pero la novedad se reconoce en las nuevas significaciones. Vale la pena observar que la incertidumbre provocada por la aparición de algo nuevo debe ser comprendida como ausencia de certeza, en términos de cálculo y de exactitud pero no como equivocidad. Asimismo, el sujeto de la historia "no crea por entero su papel: frente a situaciones típicas, toma decisiones típicas, y Nicolás II, volviendo a encontrar aun las palabras de Luis XVI, desempeña el papel ya descrito de un poder establecido frente a un poder nuevo" (Merleau-Ponty, 1945: 103). En otros términos, no hay ni en la historia pública ni en la historia personal una novedad absoluta ni una repetición continua. Y, esto sucede tanto con el acontecimiento histórico como con los acontecimientos que atañen al saber exacto. Así, por ejemplo, es conocida la descripción que Merleau-Ponty realiza acerca de los avances de la geometría. El teorema de Pitágoras puede parecer a primera vista como una entidad eterna; sin embargo, no puede pasar inadvertido el hecho de que, si bien la geometría euclidiana encierra ideas ciertas, estas ideas tienen su origen en un momento histórico determinado. La geometría del siglo XIX las retoma, las define de otra manera, y finalmente considera el espacio euclidiano como un caso particular de un espacio más general. Esto quiere decir que el espacio euclidiano en tanto formación cultural, está ligado a un cierto estado del saber, que con posterioridad será retomado o sublimado por las generaciones siguientes. Así:

Decir que un acontecimiento tiene lugar, es decir que será verdad para siempre que ha tenido lugar. Cada momento del tiempo, según su misma esencia, plantea una existencia contra la que los demás momentos del tiempo nada pueden. Después de la construcción, queda adquirida la relación geométrica, incluso si olvido los detalles de la demostración, el gesto matemático funda una tradición (Merleau-Ponty, 1945; 450).

Que toda adquisición sea una adquisición para siempre le otorga un carácter de eternidad porque cada presente que se produce se hunde en el tiempo como una cuña y aspira a la eternidad, pero "la eternidad no es otro orden más allá del tiempo sino la atmósfera del tiempo" (ibid., 451). Es claro que el modelo de nuestras adquisiciones es el tiempo, en el que los acontecimientos se excluyen unos a otros pero donde cada uno adquiere un lugar intransferible. Así, por ejemplo, quien ha tenido la posibilidad de contemplar una determinada pintura sabe que ésta se ha instalado en su vida para siempre y, aún en el caso en que no pueda recordar todos los detalles de lo que vio, esa experiencia estética, será para ese hombre, la de alguien que ha conocido, por ejemplo, la pintura de Van Gogh. Así, toda experiencia vivida será una adquisición irreductible y sobrevivirá al tiempo. La comunicación o la recepción de los hechos en la historia responden al modelo del tiempo:

Lo que se llama intemporal en el pensamiento es aquello que, por haber retomado lo pasado y comprometido el porvenir, presumiblemente es de todos los tiempos $y$, no es pues de ninguna manera trascendente al tiempo. Lo intemporal es lo adquirido (Merleau-Ponty, 1945:450). 


\section{3 La contingencia del acontecimiento}

Para Merleau-Ponty, el rasgo decisivo que está en juego en el acontecimiento es la contingencia. El acontecimiento permite ver que no hay razón de ser última para "lo que llega"; que, si bien hay sentido, puesto que las orientaciones tomadas en la historia son motivadas, no hay un sentido que aparece como desarrollo de la historia. Tomemos dos ejemplos dados por el autor, para ilustrar en qué medida el acontecimiento está marcado por la contingencia. En la Fenomenología de la percepción, al referirse a la descripción que Stendhal realiza acerca de la batalla de Waterloo (llamativamente Marion utiliza el mismo ejemplo para caracterizar el acontecimiento histórico), ${ }_{2}^{2}$ se deja en claro que el acontecimiento no puede ser considerado como un objeto. Así, el historiador que pretende observar lo que sucede, observa la batalla con una mirada de sobrevuelo, esto es, como un espectáculo y la ve desde todas partes, reúne una multitud de testimonios que le permiten saber, incluso, cómo terminó. De esta manera, cree captarla en su verdad, pero lo que capta es una representación. En palabras de Merleau-Ponty, "Fabrice quería ver la batalla de Waterloo como se ve un paisaje y solo encuentra episodios confusos, que no permiten captar la batalla y menos predecir su final porque el momento en que finaliza es contingente" (Merleau-Ponty, 1945: 416). La contingencia no debe ser interpretada como sinónimo de irracionalidad porque siempre se espera que alguna posibilidad se realice y las posibilidades se van delineando en el transcurso de los hechos. Esto significa que el acontecimiento no es previsible pero la historia deja entrever que algo sucederá. Asimismo, en el ensayo "La guerra tuvo lugar", al hilo de sus reflexiones sobre la guerra y el antisemitismo, Merleau-Ponty observa que la pasión va acompañada de un elemento de azar que es reabsorbido, por ejemplo, en el caso del amor por el encuentro con la persona que se va a amar. Este encuentro está de alguna manera prefigurado por el pasado y una vez que acontece activa una serie de posibilidades que sin él se hubieran adormecido. Una vez que el amor se realiza toma la figura de un destino, pero "el día del primer encuentro es absolutamente contingente" (Merleau-Ponty; 1963: 253). A través de esta descripción Merleau-Ponty extrae una serie de conclusiones que son atinentes a la historia. En primer lugar, el acontecimiento histórico no puede ser explicado totalmente, solo pueden indicarse las motivaciones que dibujan una historia posible. Por otro lado, los hombres se presentan unos a otros sobre un fondo de historicidad que no han escogido pero que, en cierto sentido, les asigna un rol que los excede, y que modifica el sentido de cada una de sus acciones. En otros términos, el movimiento de la historia hace visible que la contingencia no significa un destino oscuro o una fatalidad impenetrable, no existe una fuerza o un genio maligno que dirige la vida humana hacia el caos porque cada una de nuestras acciones, al tener en cuenta las otras, las recobra orientándolas hacia un sentido universal: "el mundo humano es un sistema abierto o inacabado y la misma contingencia fundamental que lo amenaza de discordancia lo sustrae también a la fatalidad del desorden" (Merleau-Ponty, 1960: 304).

A partir de aquí, podemos preguntarnos ¿cómo hay que pensar la historia para poder dar cuenta tanto del sentido como de la fragilidad del acontecimiento interhumano? Si la historia es el lugar de "nuestras interrogaciones y de nuestros asombros" (Merleau-Ponty, 1960: 88) y si es lícito hablar de un enigma en la historia es porque una sobreabundancia de sentido y una riqueza de lo implícito se enlazan en la iniciativa

\footnotetext{
2 Llama la atención que Jean-Luc Marion utiliza el mismo ejemplo para caracterizar al acontecimiento histórico, que describe en los siguientes términos: “(..) la batalla sucede y sucede sola, sin nadie que la haga estructuralmente hablando ni la decida, la batalla sucede y cada uno la ve suceder, perfilarse y desaparecer tal como había llegado, es decir, a partir de ella misma. En el hacerse de la historia (Geschichte), la batalla se hace a partir de sí misma, a partir de un punto de vista que solo ella permite unificar, sin ningún horizonte único. (...) Por lo tanto, en la historia que se cuenta (Historie), la batalla requerirá que se adicionen los horizontes en un número (horizontes conceptuales) indefinido: horizonte militar (...), horizonte diplomático, político, económico, ideológico etc.". Marion comenta que: "esta pluralidad de horizontes impide prácticamente constituir el acontecimiento histórico en un objeto e impone adoptar una hermenéutica sin fin en el tiempo" (Marion, 319: 1997).
} 
humana. Años más tarde, Merleau-Ponty expresa con claridad sus primeras intuiciones acerca de la comprensión de la historia: la pregunta por el sentido de la historia tiene presente que es un hecho irrefutable que son los hombres los que hacen la historia, pero también es un hecho irrefutable que hay un sentido que no depende de la voluntad de los hombres. Con otras palabras, más allá de lo que los hombres pueden producir, "hay sentido, dimensiones, figuras", y, sin embargo, "son hombres los que hablan, piensan, ven" (Merleau-Ponty, 1960: 28). Estas dos instancias que no implican una contradicción, ponen de manifiesto el carácter paradójico de la historia. Por una parte, la historia se nos volvería incomprensible si ella no estuviera recorrida por un sentido, por la otra, la historicidad misma correría el riesgo de desaparecer si la historia dejará de ser una aventura imprevisible.

\section{Conclusiones}

A modo de conclusión, quisiera señalar la vigencia de las reflexiones de Merleau-Ponty en la época de Fenomenología de la percepción en vistas a un diálogo posible dentro de las discusiones contemporáneas acerca del acontecimiento. Intentaré esbozar en lo que sigue algunas consideraciones actuales en este debate, con la finalidad de destacar encuentros y desencuentros entre aquéllas y la historicidad del acontecimiento elaborada por el fenomenólogo francés expuesta a lo largo del trabajo.

Un enfoque fenomenológico del acontecimiento como génesis de sentido puede señalarse en la Nueva Fenomenología en Francia (Tengelyi et al, 2011). Según Hans-Dieter Gondek y László Tengelyi, una de las cuestiones compartidas por autores como JeanLuc Marion, Michel Henry o Marc Richir, es la del acontecimiento comprendido como acontecimiento de sentido (Sinnereignisse). Sin embargo, desde estas perspectivas, que destacan la radicalidad del acontecimiento en cuanto a su aparecer, no es posible avanzar en su carácter histórico y temporal y, particularmente, en lo que, desde el punto de vista de la fenomenología clásica, se denomina su "génesis de sentido". En este punto comparto, a modo de ilustración del problema, la sagaz objeción de Claudia Serban, quien afirma: "uno puede preguntarse si para esta fenomenología, que comienza a hablar de lo acontecial del fenómeno, se puede pasar por alto la referencia a la historia. Si esto es así, puede resultar problemático cortar radicalmente el pensamiento del acontecimiento del pensamiento de la historia" (Serban, 2017: 136).

En este sentido, la aparición repentina y abrupta del acontecimiento, tal como la piensa, por ejemplo, F. Dastur, nos resulta problemática en la medida en que corta radicalmente el acontecimiento de su historicidad. La dificultad que proviene del carácter súbito del acontecimiento, que lo desliga de su temporalidad, es señalada de manera paradigmática por Merleau-Ponty, quien afirma que: “[...] habría ilusión prospectiva en detener el presente en el umbral de un porvenir vacío como si cada presente no se prolongará hacia un horizonte de futuro [...]" (Merleau-Ponty, 1968: 45).

En la dirección de la historicidad del acontecimiento merece la pena destacar las reflexiones de Paul Ricoeur, quien ha dedicado alguno de sus ensayos al estudio de este fenómeno y que, en ciertos aspectos, confluye con la posición fenomenológica de Merleau-Ponty. Sin ninguna duda, el acontecimiento aparece como novedoso, pero su aparición no es independiente de la tríada "acontecimiento, estructura y coyuntura". Según Ricoeur, la interpretación del acontecimiento, que se aleja de la Escuela de los Anales, recupera las estructuras y coyunturas inestables dando lugar "a lo imprevisible, a lo contingente" (Ricoeur, 1992: 31). Desde su perspectiva, un acontecimiento es considerado como histórico cuando cumple tres condiciones: 1 ) son hombres los que hacen llegar algo o son afectados por los acontecimientos que llegan u otros hacen llegar, 2) los acontecimientos deben ser lo suficientemente interesante para que los 
testigos oculares los registren y 3) esta última condición pone en juego la "puesta en intriga" que produce un desfasaje entre el acontecimiento tal como sobreviene y el acontecimiento tal como es contado o relatado (cf. Ricoeur, 1992: 31).

Por otra parte, el acontecimiento sobreviene en un campo rodeado de horizontes: el de lo instituido y el de las múltiples posibilidades que el acontecimiento engendra. Puede destacarse que ambos filósofos acuerdan en tratar al acontecimiento afectado tanto por sus huellas como por la tradición: "entre la huella dejada y recorrida, y la tradición transmitida y recibida, se revela una afinidad profunda" (Ricoeur, 1985:331). Mientras que la huella hace referencia a la materialidad de la marca, es decir, a "su inscripción en el tiempo del universo", la tradición alude a otro tipo de exterioridad: "la de nuestro ser afectados por un pasado que nosotros no hemos hecho" (Ricoeur, 1985: 331). En la misma línea, Merleau-Ponty sostiene que la sedimentación es: "huella de lo olvidado y por lo mismo llamado a un pensamiento que cuenta con él y va más lejos" (Merleau-Ponty, 2003: 99).

Lo asombroso de la mirada ricoeuriana sobre el retorno al acontecimiento reside en ver que la historiografía no tiene "el monopolio del acontecimiento", el que no solo concierne a los historiadores sino también a los hombres que hacen y padecen la historia. El acontecimiento tiene lugar en la constitución misma del tiempo histórico, donde se conjugan "la memoria de lo que fue, la expectativa de lo que será y el surgimiento presente de lo que hacemos y padecemos como agentes y pacientes de la historia" (Ricoeur, 1992:35). De esto modo, si el acontecimiento es inatacable en el plano de la historiografía, es porque transcurre "en el plano de la historia efectiva que hacemos y padecemos" (Ricoeur, 1992:35). En virtud de ello, el acontecimiento reúne las dos significaciones de la palabra historia: "serie de los acontecimientos en vías de producirse, y relato de los acontecimientos por aquellos que no lo han vivido y los reconstruyen" (Ricoeur, 1992:35).

Finalmente, Ricouer piensa el entrelazamiento entre acontecimiento e historia a partir de una "dialéctica abierta". Explícitamente, en el apartado titulado: "Hacia una hermenéutica de la conciencia histórica", afirma que, ante la imposibilidad de "una mediación total", solo queda la posibilidad de "una mediación abierta, inacabada, imperfecta, una red de perspectivas cruzadas entre la espera del futuro, la recepción del pasado, la vivencia del presente, sin Aufhebung en una totalidad en la que coincidirían la razón de la historia y su efectividad" (Ricoeur, 1985: 300). En la misma dirección, la dialéctica implica para Merleau-Ponty totalizaciones precarias que nunca se completan y que pueden reorganizarse en cada presente. Las coincidencias con el autor de la Fenomenología de la percepción, resultan, una vez más, asombrosas.

En lo que concierne a los historiadores, podemos señalar el sugerente ensayo de Marlène Zarader titulado "El acontecimiento entre fenomenología e historia", que ofrece un panorama de las posiciones fenomenológicas acerca del acontecimiento y la manera en que los historiadores lo conciben. En lo que respecta a las posiciones fenomenológicas distingue dos registros: 1) el primero, representado por "el fenómeno-acontecimiento": se trata de aquellos fenómenos "intimos" como un duelo o una enfermedad o "colectivos" como la caída de las torres gemelas. Según la autora, tales acontecimientos tienen lugar en el orden óntico, en el que los acontecimientos se presentan como impuestos a los sujetos que lo viven (cf. Zarader, 2004: 290). 2) En lo que atañe al segundo registro que aborda el "acontecimiento como origen de los fenómenos" (Zarader, 2004: 299), se intenta interrogar al acontecimiento en vistas de una redefinición de la "fenomenalidad como tal" (Zarader, 2004: 299). Más precisamente, se trata de dos versiones de la fenomenología: una, orientada a describir "el fenómeno-acontecimiento", de clara cepa husserliana; la otra, que hace del acontecimiento "el origen de los fenómenos" y, que, se corresponde con la versión 
heideggeriana de la fenomenología que "puede hacer del acontecimiento el origen de los fenómenos puesto que sitúa este origen en el carácter acontecial del ser" (ibid). Ahora bien, el interés central, según mi opinión, reside en la articulación que la autora intenta hacer "entre fenomenología e historia". De acuerdo a ello, se propone dilucidar la cuestión de saber "¿cómo el acontecimiento se inscribe en su historia?" (Zarader, 2004: 300). La autora presupone que el historiador articula, quizás sin saberlo claramente, tres maneras de relacionarse y de analizar los acontecimientos. En primer lugar, se encuentra la actitud natural que corresponde "a los actores de la historia". Más precisamente, se trata de la vivencia del acontecimiento, por parte de aquellos que lo viven, que sorprende por su capacidad de fractura y que es vivida como real. A continuación, la actitud fenomenológica describe el acontecimiento como fenómeno, sin prejuzgar nada acerca de su ser. Finalmente, la actitud hermenéutica, que mediatizada por la actitud fenomenológica, y, más allá del hecho de que "ha habido acontecimiento" permite que el historiador juzque la situación. Su intención es cualificar (qualifier) el acontecimiento de manera tal que al hacerlo supera el antes y el después y realiza el trabajo de tejer "la trama del sentido" (cf. Zarader, 2004: 309). En términos de Ricoeur, "la intencionalidad histórica...crea la cualidad histórica de la historia" (Ricoeur, 1985: 296) evitando que ella se disuelva en los saberes con los cuales trabaja la historiografía como la economía, la demografía o la etnología.

En vista a señalar las posibilidades que la fenomenología de Merleau-Ponty ofrece para este diálogo, me interesa poner de manifiesto los tres rasgos que, según la autora, podrían ser un punto de inflexión para un intercambio fecundo tanto para los fenomenólogos como para los historiadores; primeramente, la idea de singularidad: el acontecimiento que sobreviene se presenta como incomparable con algún otro. En segundo lugar, la cesura (césure), esto es, la potencia separadora por la cual una totalidad hasta entonces significativa se deshace mientras que otra se configura. $\mathrm{Y}$, finalmente, la interferencia o ruptura de inteligibilidad del sentido, que pone el sentido, momentáneamente, en suspenso (cf. Zarader, 2004: 302). Un rasgo decisivo del acontecimiento es la noción de cesura que, en términos merleau-pontyanos, es interpretada como écart, como desviación, distancia o diferencia. Así, por ejemplo, lo que el pintor aporta a la expresión del mundo es una variante que en un principio solo aparece por oposición a las otras pinturas, pero que se manifiesta no obstante en cada elemento y en el conjunto, de suerte que se trata “(...) de una distorsión sistemática dirigida por la nueva relación con el mundo" (Merleau-Ponty, 2000: 29). La aparición de algo nuevo se encuentra en cierto modo previamente trazado en una estructura como sistema abierto y comprometido en el devenir del pensamiento. La sedimentación no es el fin de la historia, pero no hay historia si algo de lo que pasa no permanece y si cada presente, justamente en su singularidad, no se inscribe en el cuadro de lo que ha sido y continúa siendo. Tal es el sentido de lo adquirido, que ya destacamos en Fenomenología de la percepción.

Finalmente, dentro de la perspectiva que aboga por la historicidad del acontecimiento, intérpretes como R. Terzi sugieren que el conjunto de los análisis de Merleau-Ponty sobre la institución esbozan un cuadro para una comprensión del acontecimiento histórico y su dinámica desde el punto de vista fenomenológico. A partir de los análisis sobre la noción de institución, Terzi establece algunas analogías con la noción de acontecimiento proveniente de los estudios historiográficos. Así, por ejemplo, por su potencia de apertura el acontecimiento llama a una serie de retomas cuyo rol principal lo cumple la memoria: desde el punto de vista de la historiografía, el acontecimiento comprendido por los historiadores como "huella significante" tiene su correlato con un "giro memorial" que analiza el rol de la memoria en relación con nuestra época y con los acontecimientos pasados (cf. Terzi, 2017: 12). Merleau-Ponty advierte esto al destacar que la institución consiste en la capacidad de dar al pasado una nueva vida que es la "forma noble de la memoria". En segundo lugar, la relación 
del acontecimiento con la memoria pone en juego una cierta manera de comprender la temporalidad, presentada por Merleau-Ponty como "modelo de la institución". La temporalidad del acontecimiento no es del orden de lo objetivo ni de lo subjetivo, sino que se trata de "una transtemporalidad originaria", que es la de "la institución en estado naciente". Hemos advertido que ya en Fenomenología de la percepción el tiempo aparece como el modelo de lo adquirido; sin embargo, no nos pasa inadvertido que las reflexiones acerca de la manera de pensar la temporalidad adquieren en los últimos escritos matices que, en cierto sentido, exigirían un tratamiento especial, y, que no es el propósito de este trabajo. A modo de preludio, recordemos el siguiente texto:

El tiempo no es envolvente ni envuelto: desde mí hacia el pasado existe cierto espesor que no está hecho de una serie de perspectivas, ni de la conciencia de la relación entre ellas, sino que es obstáculo y lazo (...) El tiempo es el modelo mismo de la institución: pasividad-actividad, continúa porque ha sido instituido; se dispara, no puede dejar de ser, es total porque es parcial, es un campo (Merleau-Ponty, 2003: 36). 


\section{Bibliografía}

"Dastur, F. (2000). Phenomenology of the Event: Waiting and Surprise, Hypatia, 15(4).

» de Certeau, M. (1994). La Prise de parole. París: Points/Seuil.

"Dosse, François (2010). Renaissance de l'événement. Un défi pour l'historien: entre Sphinx et Fénix. París: PUF.

" Marion, J. L. (1997). Étant donné. Essai d'une phénoménologie de la donation. París : PUF.

» Merleau-Ponty, M. (1945) Phénoménologie de la perception. París: Gallimard.

» Merleau-Ponty, M. (1953). Éloge de la philosophie et autres essais. París: Gallimard.

» Merleau-Ponty, M. (1955). Les aventures de la dialectique. París: Gallimard.

》Merleau-Ponty, M. (1960). Signes. París: Gallimard.

" Merleau-Ponty, M. (1964). Le visible et l'invisible. París: Gallimard.

» Merleau-Ponty, M. (1963). Sens et non-sens. París: Nagel.

" Merleau-Ponty, M. (1968). Collège de France. Résumés de cours 1952-1960. París: Gallimard.

» Merleau-Ponty, M. (2000). Parcours deux 1951-1961. Lagrasse: Verdier.

" Merleau-Ponty, M. (2003). L'institution. La passivité. Notes de cours au Collège de France (1954-1955). París: Belin.

»Ricoeur, P. (1992). Le retour de l'événement, Málanges de l'Ecole française de Rome, Italie et Mediterranée, t. 104, $n^{\circ} 1$.

》 Ricoeur, P. (1991). Événement et sens, L'espace et le temps. Actes du XII Congrès de l'Association des Sociétés de Langue Française, Dijon: Vrin.

» Ricoeur, P. (1985). Temps et récit. Le temps reconté. París: Seuil.

》 Ricoeur, P. (1985). Tiempo y narración I. Configuración del tiempo en el relato histórico. México: Siglo XXI.

»Serban, C. (2017). L'événement historique: un paradigme de la phénoménalite?, Alter, 25.

» Tengelyi, L. y Gondek, H. D. (2011). Neue Phänomenologie in Frankreich. Fráncfort: Suhrkamp.

" Terzi, R. (2017). Institution, événement et histoire chez Merleau-Ponty, Bulletin d'analyse phénoménologique, XIII 3.

» Terzi, R. (2016). Événement, champ, trace: le concept phénoménologique d'institution, Philosophie, 4.

» Thierry, Y. (2005). L'institution et l'événement selon Merleau-Ponty, en Bonan, Ronald (ed.), Merleau-Ponty: de la perception à l'action. Aix-en-Provence: Publications de l'Université de Provence.

»Zarader, M. (2004). L'evénement, entre phénoménologie et histoire, Tijdschrift voor Filosofie, 66ste Jaarg., Nro 2, Haag: Peeters Publishers. 
